\title{
Constitutional Obesity v/s Acquired Obesity and Their Cardiovascular Risks
}

\author{
Tarun Saxena ${ }^{1 *}$ Azeema Ozefa Ali $^{1}$ and Manjari Saxena ${ }^{2}$ \\ ${ }^{1}$ Department of Internal Medicine, Mittal Hospital and research centre, India \\ ${ }^{2}$ Department Yoga and Physical education, Mittal Hospital and research centre, India
}

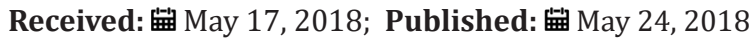

*Corresponding author: Tarun Saxena, Department of Internal Medicine, Mittal Hospital and research centre, India

\section{Introduction}

Obesity is a worldwide increasing problem. It is observed in the medical outdoor that certain people who by definition of obesity (BMI) are obese but do not have any obesity related medical diseases. Is there any discrepancy in the obesity of these people from the others? This review article has tried to answer such questions.

\section{Definition/Measurement}

Obesity is defined as BMI $1>30 \mathrm{~kg} / \mathrm{m} 2$ or $20 \%$ increase in body weight than the ideal body weight [1]. Normal BMI is supposed to be in between $18.5-25 \mathrm{~kg} / \mathrm{m} 2$. Obesity is also measured by various other means, skin fold thickness, waist circumference (WC), waist-to-hip ratio, or by assessing visceral adiposity by imaging techniques. Obesity is considered as important risk factor besides other risk factors for various cardiovascular diseases.

\section{Types of Obesity}

\section{Constitutional}

Various factors associated with constitutional obesity are

a) Genetic: Associated with genetic mutations, the effect of mutations on the obesity phenotype being amplified by the development of obesity producing environment.

b) Racial/ethnic/territorial: Various races/ ethnic and territorial regions have obesity. One common example is nonHispanic Blacks have the highest obesity level, followed by Hispanics, then non-Hispanic Whites, and lastly non-Hispanic Asians [2].

c) Gender: Females are at higher risk of developing morbid obesity than males. This discrepancy is explained partly by female-specific genetic associations or by stronger effect sizes of genetic variants in females. d) High birth weight: Common finding these days. Baby born with high birth weight possibly have excess fat cells, there are data available showing early-life influences, beginning with the intrauterine environment and continuing through the first few years of life, affect body fatness throughout the life course [3].

e) Constitutionally obese persons do not have diseases commonly considered to be associated with obesity e.g. Hypertension, ischemic Heart disease, diabetes etc. [4].

\section{Pathological/acquired obesity}

Obesity which is acquired as a result sedentary life, irregular dietary habits, lack of exercise, and central obesity due to chronic mental stress and mental exhaustion. Chronic stress increases basal sympathetic discharge produces insulin resistance and tooth pick obesity. This obesity is better measured by waist-hip ratio. Such obesity increases the risk of hypertension, dyslipidaemia, and atherosclerosis and type- 2 diabetes [5]. It creates a proinflammatory state, and a pro-thrombotic state; thus leading to cardiovascular diseases. Constitutional obesity is considered to grow from infancy which is again sometimes difficult to differentiate with pathological obesity creating clinical problem for the physicians that which obese should be treated?

\section{Clinical implications}

Constitutional Obesity:

a) Constitutional obesity is different from acquired/ pathological obesity

b) It is difficult to reduce weight in persons with constitutional obesity and to maintain at lower levels for longer period is even more difficult. 
c) Constitutional obesity carries less risk for cardiovascular diseases than acquired /pathological obesity

d) Persons with constitutional obesity have some inherent protective mechanism which prevents them from various cardiovascular diseases in absence of other risk factors

e) These people require reassurance only, and no therapy?

Pathological obesity:

a) Pathological obesity carries a definite risk for cardiovascular and other diseases

b) Pathological obesity must be treated

c) It requires adequate diet and exercise management

d) Most importantly, to reduce central obesity, adequate mental rest, sound sleep for 7- 8 hours, spending some time in garden, and practicing some relaxation techniques is important [6].

\section{Conclusion}

All efforts must be done to identify constitutional versus pathological obesity; as there is a difference in management of both types. On one hand constitutional obesity requires no special effort; whereas, on the other hand pathological obesity requires definite treatment. There should be emphasis to reduce stress/mental exhaustion in pathological obesity which is the prime reason for such obesity.

\section{References}

1. Obesity-Wikipedia (https://en.m.wikipedia.org>wiki>obesity).

2. Forrest KYZ, Leeds MJ, Ufelle AC (2017) Epidemiology of obesity in the Hispanic Adult Population in the United States 40(4): 291-297.

3. Cnattingius S, Villamor E, Lagerros YT, Wikström AK, Granath F (2012) High birth weight and obesity- a vicious circle across generations. Int J Obes (Lond) 36(10): 1320-1324

4. Undurti N (2007) DasObesity and its relationship to coronary heart disease. Eur heartj 28(23): 2953-2954.

5. Tarun Kumar Saxena, sanjiv Maheshwari (2014) Aetio pathogenesis of Type-2 Diabetes Mellitus: Could chronic stess play an Important Role? p. 62.

6. Saxena T, Saxena M (2016) Possible Clinical Implications of High Left Ventricular Ejection force and exaggerated Sympathetic skin response In Hypertensive Patients. Ann ClinExp Hypertension 4(1): 1035.
This work is licensed under Creative Commons Attribution 4.0 License

To Submit Your Article Click Here:

Submit Article

DOI: 10.32474/ACR.2018.01.000107

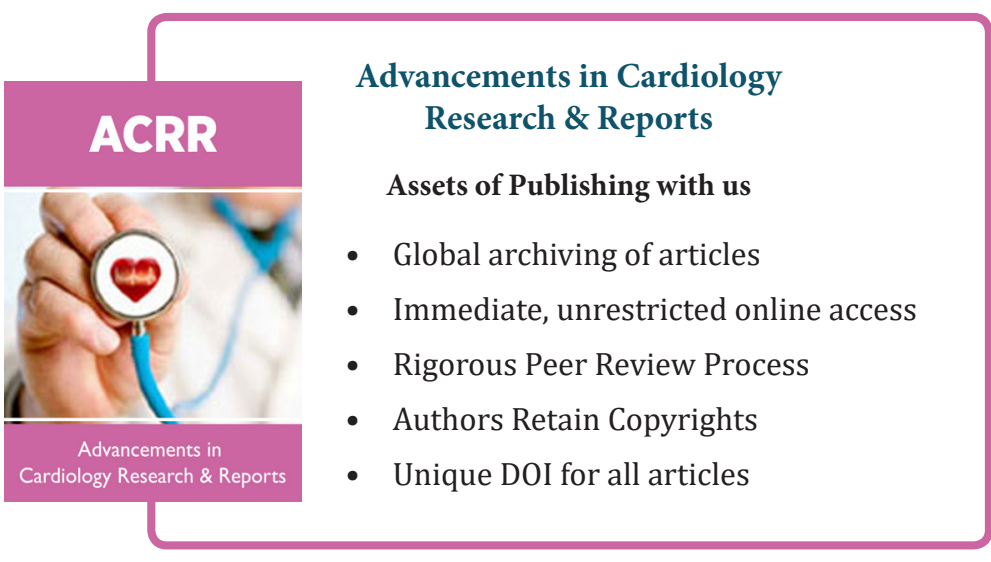

